Titre: Le hors-nORME À MAYOTTE. PROduCtions ÉCRITES SUR FACEBOOK ET SUR LES PANNEAUX PUBLICITAIRES

Auteur(s): LAVIE Maturafi, Doctorante, Praxiling, UMR 5267, UniVersité Paul Valéry MontPellier 3-CNRS

PUBLICATION: ÉCRITS HORS-NORMES

PAGES: $65-78$

Directeurs: Agnès Steuckardt et Karine Collette

ÉdITEUR: LES ÉDITIONS DE L'UNIVERSITÉ DE SHERBROOKE, 2019

ISBN: 978-2-7622-0360-8

URI: HTTP://HDL.HANDLE.NET/11143/15573

DOI: HTTPS://DOI.ORG/10.17118/11143/15573 


\section{Le hors-norme à Mayotte. Productions écrites sur Facebook et sur les panneaux publicitaires}

Lavie Maturafi, Doctorante, Praxiling, UMR 5267, Universite̋ Paul Valéry Montpellier 3-CNRS

Résumé : L'objectif de cette recherche est de montrer comment les discordances à la norme graphique du français et à la norme graphique non stabilisée du shimaoré produisent un écrit hors-normes à Mayotte. Pour ce faire, une étude a été menée sur les écrits présents sur les panneaux publicitaires à Mayotte ainsi que sur ceux produits par des scripteurs mahorais sur le réseau social Facebook. Nos résultats montrent qu'il parait possible de distinguer un shimaoré francisé, influencé par les règles phonogrammiques du français qui est notamment présent sur les panneaux publicitaires et sur Facebook et un shimaoré bantu, inspiré du système des langues bantu, pour les écrits institutionnels issus des tentatives, encore inabouties, d'instituer une norme écrite.

Mots-clés : shimaoré, français, cohabitation linguistique, discours hors-norme, Facebook, panneau publicitaire.

Abstract : The aim of this research is to show how discordances between the French graphic standard and the Shimaore graphic standard produce a writing which is out of the norm in Mayotte. To carry this out, we study writings shown on billboards at Mayotte and messages in Shimaore present on the Facebook social network. Our results show that it is possible to distinguish a Shimaore influenced by the phonogrammic rules of French and a Shimaore inspired by the bantu languages for the institutional writings.

Key words : shimaore, french, linguistic cohabitation, speech out-standard, Facebook, billboard. 
Mayotte est une île faisant partie de l'archipel des Comores. Ce territoire est français depuis 1841, année de cession de l'île à la France ; il est officiellement devenu un Département français en 2011. De nombreuses langues y sont parlées, parmi lesquelles le shimaoré et le français.

Signalons que le statut de ces deux langues n'est pas le même sur l'île : selon Cassagnaud (2007 : 212), «le français est la langue de l'école, de l'enseignement et de l'écrit, jusqu'à l'environnement spatial comme les panneaux publicitaires, les noms des rues qui commencent à apparaître alors que l'espace culturel occupé par les français est incertain ».

Parallèlement à cela, le shimaoré est, d'après Rombi (1983 :19-20), « un parler bantu de l'île de Mayotte, microcosme culturel et linguistique, point de rencontre et de fusion des mondes africains, arabo-persan et malgache [...] [qui] appartient au comorien qui se divise en deux groupes dialectaux distincts : anjouannais-mahorais, grand-comorien-mohélien ». Le shimaoré est usité, de manière non officielle, dans les mêmes contextes que le français, non seulement à l'oral (médias, justice, politique, etc.), mais également dans les pratiques écrites ordinaires (panneaux publicitaires, affichette, etc.), ou émergentes (forum, réseaux sociaux).

La cohabitation linguistique les deux langues évoquées génère des transferts sur le plan graphique en shimaoré et facilite le code-switching. Différentes raisons peuvent expliquer ce phénomène, comme:

- la volonté de vouloir écrire dans sa langue maternelle malgré le fait quel soit spécifiquement orale. C'est la raison pour laquelle les Mahorais calquent leur écrit sur le français qui est, avec l'arabe, l'une des langues écrites connues et apprises sur l'île ;

- le souhait de spécifier son identité linguistique en montrant qu'on maîtrise telle ou telle langue ;

- le développement des nouveaux supports tels que les SMS ou les réseaux sociaux qui ont favorisé ces pratiques langagières.

L'objectif de cette étude est de montrer comment les discordances à la norme graphique instituée du français et à la norme graphique non stabilisée du shimaoré produisent un écrit hors-normes à Mayotte. Notre hypothèse est que la non-codification d'un alphabet fixe du shimaoré provoquerait la coexistence de deux codes écrits pour le mahorais, à savoir :

- $\quad$ une écriture institutionnelle : shimaoré bantu (inspiré du système des langues bantu)

- $\quad$ une écriture spontanée : shimaoré francisé (avec les caractéristiques du français).

Pour mener à bien notre étude, nous présenterons les diverses tentatives de mise à l'écrit du shimaoré, puis nous verrons en quoi ces tentatives divergent par rapport aux écrits produits sur les panneaux publicitaires et sur Facebook.

\section{Tentatives de mise à l'écrit du shimaoré}

Si le mahorais est avant tout une langue orale, des tentatives de mise à l'écrit ont été opérées : le Groupe de Recherche sur le Plurilinguisme à Mayotte (GRPM), l'Association SHImaoré MEthodique (SHIME), le linguistique Haladi Madi, entre autres, ont œuvré à la création d'un alphabet fixe et à l'édification de normes à l'écrit. 


\section{La question de l'alphabet: les points d'accords}

La question de la transcription des langues à Mayotte est au cœur des préoccupations depuis quelques années. Si, par le passé, la mise à l'écrit concernait une minorité de groupes et quelques passionnés des langues, aujourd'hui, il s'agit de l'affaire de tous les Mahorais.

En janvier 2006, lors du colloque La Marche de Mayotte vers le droit commun, organisé par le Conseil Général à Mamoudzou, « une divergence entre le système proposé par Haladi Madi, et celui proposé par le comité des langues créé par le CCEEM (Conseil de la Culture, de l'Éducation et de l'environnement de Mayotte) en août 2005 » a pu être relevée (Laroussi : 2009a : 29).

Si, lors de ce colloque, l'alphabet élaboré par le linguistique Haladi Madi a été distribué, le débat sur la fixation du shimaoré sera relancé deux mois plus tard, plus précisément en mars 2006, lors du colloque «Bilinguisme et interculturalité à Mayotte pour un aménagement du système éducatif », organisé par le vice-rectorat de Mayotte, l'IRD (Institut de Recherche pour le développement) et soutenu par le Conseil général de Mayotte. Lors de cette manifestation, diverses propositions ont été formulées pour grammatiser les langues mahoraises. Depuis, aucun organisme officiel ne s'est prononcé sur la question malgré de nombreuses discussions autour de la question de la transcription des langues. Il y a eu cependant consensus à présent d'un certain nombre de signes graphiques.

Les voyelles

\begin{tabular}{|l|l|l|}
\hline \multicolumn{1}{|c|}{ Les voyelles orales } & Les semi-voyelles & La nasalisation phonétique \\
\hline $\mathrm{a}:$ gari (voiture) $:$ hale (conte) & $\mathrm{y}$ : haya (honte) & â : âlama (marque, trace) \\
$\mathrm{i}:$ : ini (quand) & & \\
$\mathrm{o}:$ moro (feu) & & \\
$\mathrm{u}:$ putu (piment) & & \\
\hline
\end{tabular}

Les voyelles orales "a", "i" et "o" se prononcent comme en français. Alors que le "e" et le "u" se prononcent distinctement:

• e" se dit "é" comme dans pare (« route ») (prononcé paré),

- "u" se dit "ou" comme dans putu («piment») (prononcé poutou).

Concernant les semi-voyelles "y" et "w" :

- le "y" mahorais se prononce comme celui du français dans le verbe payer, c'est le cas par exemple du substantif haya (« honte »);

- le "w" en shimaoré se dit comme celui de l'anglais dans le mot world (« monde »), comme par exemple dans le nom commun wana (" enfants»).

Dans certains contextes, le mahorais a tendance à nasaliser les voyelles. Cette nasalisation est marquée graphiquement par un accent circonflexe. Cependant, selon Madi : 
Le mahorais n'atteste pas phonologiquement des voyelles nasales. Nous avons en revanche un phénomène de nasalisation phonétique lié au contexte. En effet, à chaque fois qu'une voyelle orale se trouve précédé du son consonantique identifié en shimaoré comme [§], elle se nasalise systématiquement [...]. Ce son consonantique [\$] qui nasalise systématiquement en shimaoré les voyelles qui le succèdent, que ça soit dans les items empruntés à l'arabe ou au français, nous provient de l'arabe. Mais, ce qui est frappant, c'est que ce son n'est nasalisé en arabe standard qui et la langue du coran dont les Mahorais s'initient dès leur jeune âge. (2005: 38).

C'est, d'après Madi, la consonne [G] empruntée à la langue arabe, qui, dans certains cas, nasalise les voyelles qu'elle suit. La voyelle "a” au contact de cette consonne devient une voyelle nasale. C'est le cas par exemples des mots : âviyo («avion ») prononcé [Gãvijo], âlama ( signe », « marque », « trace ») prononcé [ৎãlàma], etc.

\section{Les consonnes}

Les consonnes simples

\begin{tabular}{|l|l|l|}
\hline Les consonnes simples & $f:$ furaha (joie) & $n:$ nane (huit) \\
$g:$ gashi (pétrole) & $p:$ papa (requin) \\
$\mathrm{h}:$ hale (conte) & $\mathrm{r}:$ range (peinture) \\
$\mathrm{j}:$ jana (hier) & $\mathrm{s}:$ sahani (assiette) \\
$\mathrm{k}:$ kara (nid) & $\mathrm{t}:$ titi (petit) \\
& $\mathrm{v}:$ layini (lisse) \\
& $\mathrm{m}:$ mama (pluie) \\
\end{tabular}

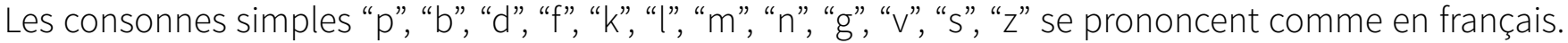
Cependant, il faut noter que le " $m$ ” et le " $n$ ” lorsqu'ils précèdent une consonne se prononcent, selon le linguiste Ahmed-Changama, «à peu près à la manière marseillaise : tro-mper, to-mber, te-ntacule, a-ndouille, ...» $1993: 17)$.

Elles peuvent aussi être utilisées comme préfixe de classe et, dans ce cas-là, elles deviennent des syllabes autonomes: on parle alors de consonnes syllabiques. En voici quelques exemples:

- le “m” : mtsi (« pilon»), prononcé m-tsi [mtsi];

- le "n": nne («quatre»), prononcé n-né [nne];

- le "h" est aspiré comme dans hale (« conte») et se prononce halé [hale].

- Le "j" se prononce comme le "j” en français dans le mot “jour”, exemple jana («hier »), prononcé jana [zana]. 
Le "r" est toujours roulé comme "r" espagnol de paro ("chômage », « arrêt »), par exemple rahisi (« moins cher») est prononcé rahissi [rahisi].

\section{Les consonnes combinées}

Les consonnes "ny", "sh", "ts" "dz" et "tsh" se prononcent comme en français, en voici quelques exemples

\begin{tabular}{|l|l|l|}
\hline \multicolumn{1}{|c|}{ Consonnes } & \multicolumn{1}{|c|}{ Exemples en shimaoré } & Correspondance en français \\
\hline ny & nyama (viande) & "gn": campagne \\
\hline sh & shahula (nouriture) & $\begin{array}{c}\text { "ch" : chat ou en anglais dans } \\
\text { "shoe" }\end{array}$ \\
\hline ts & tsano (cinq) & mouche tsé-tsé \\
\hline dz & dzina (prénom) & Dzaoudzi \\
\hline tsh & tshora (flèche, lance) & "tch": caoutchouc \\
\hline
\end{tabular}

Les consonnes "th", "dh”, "tr", "dr” et "dj” sont prononcées comme en anglais, par exemple :

\begin{tabular}{|l|l|l|}
\hline \multicolumn{1}{|c|}{ Consonnes } & \multicolumn{1}{|c|}{ Exemples en shimaoré } & Correspondance en anglais \\
\hline th & thamani (prix) & think (penser) \\
\hline$d h$ & dharuba (tempête) & other (autre) \\
\hline$t r$ & trovi (banane) & tree (arbre) \\
\hline$d r$ & dradraka (crabe) & drive (conduire) \\
\hline$d j$ & djini (esprit) & just (juste) \\
\hline
\end{tabular}

Les consonnes bi-phonématiques

Les groupes consonantiques sont des consonnes bi-phonématiques pré-nasalisées ou vélarisées. Selon Madi (2005: 65-75), il est préférable de considérer les consonnes suivantes comme étant bi-phonématiques pour des raisons de clarté et de simplification et non monophonématiques comme on le trouve dans diverses analyses linguistiques:

\begin{tabular}{|c|c|c|}
\hline $\begin{array}{l}\text { Les consonnes } \\
\text { bi-phonématiques }\end{array}$ & $\begin{array}{l}\text { mb : mbu (moustique) } \\
\text { nts : nyanstole (oiseau) } \\
\text { nd: ndovu (éléphant) } \\
\text { ndr : ndrimu (citron) } \\
\text { ndz : ndzi (mouche) } \\
\text { ng: ngama (trou) } \\
\text { pw : pwera (goyave) } \\
\text { bw : bwiri (diarrhée) }\end{array}$ & $\begin{array}{l}\text { bvw : bvwa (il y a) } \\
\text { nw: unwa (boire) } \\
\text { Iw : unolwa (être bu) } \\
\text { rw : urwa (piler) } \\
\text { sw : swiha (santé) } \\
\text { kw: kwaheri (au revoir) } \\
\text { gw : gwena (khôl) }\end{array}$ \\
\hline
\end{tabular}


Si tous les graphèmes examinés jusqu'ici font l'objet d'un consensus, quelques signes graphiques empêchent d'arriver à un accord général.

\section{Les graphèmes à l'origine du désaccord}

Comme nous l'avons vu précédemment, l'édification d'un alphabet fixe pour le mahorais engendre des divergences sur la correspondance phonogrammique à adopter pour décrire certains sons. En effet, les divergences résident dans la graphie à accorder aux explosives, aux implosives, aux sons nasalisés et à la fricative bilabiale sonore. Le tableau ci-dessous regroupe les cinq signes à l'origine du désaccord en indiquant pour chaque graphème, la graphie proposée par les différents organismes:

\begin{tabular}{|c|l|l|l|l|}
\hline \multicolumn{2}{|l|}{} & $\begin{array}{c}\text { Graphie proposée } \\
\text { par SHIME }\end{array}$ & $\begin{array}{l}\text { Graphie proposée } \\
\text { par Haladi Madi }\end{array}$ & $\begin{array}{c}\text { Graphie proposée } \\
\text { par le GRPM }\end{array}$ \\
\hline Explosives & {$[\mathrm{b}]$} & $\mathrm{b}$ & $\mathrm{b}$ & $\mathrm{bb}$ \\
& {$[\mathrm{d}]$} & $\mathrm{d}$ & $\mathrm{d}$ & $\mathrm{dd}$ \\
\hline Implosives & {$[\mathrm{b}]$} & $\mathrm{b}$ & $\mathrm{bb}$ & $\mathrm{b}$ \\
\hline $\begin{array}{c}\text { Fricative bilabiale } \\
\text { sonore } \\
{[\mathrm{d}]}\end{array}$ & {$[\mathrm{B}]$} & $\mathrm{d}$ & $\mathrm{dd}$ & $\mathrm{d}$ \\
\hline $\begin{array}{c}\text { Occlusive palatale } \\
\text { sonore }\end{array}$ & {$[f]$} & $\overline{\mathrm{v}}\left(2006^{1}\right)$ & $\mathrm{bv}$ & $\mathrm{bv}$ \\
\hline $\begin{array}{c}\text { Nasalisation } \\
\text { phonétique }\end{array}$ & {$[\mathrm{G} i]$} & $\mathrm{vh}\left(2012^{2}\right)$ & & $\mathrm{dy}$ \\
\hline
\end{tabular}

1. Association SHIME, Apprenons le shimaoré, narifundrihe shimaore, 2006.

2. Association SHIME, Apprenons le shimaoré, narifundrihe shimaore, 2012, réédition.

\section{La graphie proposée par l'association SHIME}

SHIME (SHImaoré MEthodique) est une association créée en 1998 qui est composée de chercheurs en linguistique, de formateurs, d'instituteurs shimaorophones et kibushiphones. Son objectif est de proposer une graphie stable pour les langues de Mayotte. Elle collabore notamment avec le CCEEM (Conseil de la Culture, de l'Éducation et de l'Environnement de Mayotte).

Selon Spelo, le président de l'association SHIME :

Ces graphèmes, l'association ne les a pas inventés. Des recherches [...] ont permis de constater que bien des pays du continent africain les utilisent [...]. L'appartenance du shimaoré à la grande famille des langues africaines n'étant plus à démontrer, on comprend alors facilement notre choix de nous tourner vers l'existant afin de mieux asseoir notre réflexion pour une proposition de graphie pour les langues de Mayotte. Mais le choix de chaque graphème repose aussi sur des critères bien spécifiques. (2012 : 169-170). 
S'agissant des implosives "b et d ", l'association a opté pour des lettres crossées dans le but de simplifier la lecture et l'écriture du shimaoré. Ce choix est aussi motivé, selon Spelo, par le fait que ces graphèmes sont sollicités par de nombreuses langues bantu pour le même son. L'association s'appuie également sur le résultat des tests réalisés en 1999 par l'association elle-même en collaboration avec l'IAF (Institut de recherche sur l'Apprentissage du Français), qui classent ces graphies parmi les premiers choix des Mahorais.

Pour ce qui est de la fricative bilabiale sonore, "V̄/vh", les tests menés par SHIME en 1999 auprès des Mahorais, avaient classé le v surmonté d'un tiret (pour le distinguer du v normal) parmi les premiers choix. Cependant, d'après le président de l'association, ce symbole n'existant pas dans les polices Unicode, il est plus compliqué de le taper sur un clavier d'ordinateur, d'où son remplacement par le vh, qui arrivait en deuxième choix dans les tests.

Concernant l'occlusive palatale sonore "dy", « nous (l'association) estimons que sa différence phonique claire qui le distingue du [d] doit être marquée. Nous avons donc choisi pour cela, un d" (et non un d) suivi d'un y pour le matérialiser. » (Spelo, 2012 : 170).

Le choix de marquer la voyelle nasalisée " ẽ " avec un tilde se justifie selon SHIME par le fait que dans l'alphabet phonétique international, ce signe diacritique est la marque standard pour représenter la nasalisation; de même, il s'agit du symbole le plus sollicité par les autres langues pour signaler une nasalisation. Enfin, il est désormais possible et facile d'écrire le tilde à l'aide d'un clavier standard.

\section{La graphie proposée par le linguiste Haladi Madi}

Nous n'avons pas trouvé de livre ou d’article écrit par Haladi Madi lui-même. Toutefois, en 2009, est paru le livre Mayotte, une ile plurilingue en mutation sous la direction Foued Laroussi, où l'on trouve deux articles, l'un étant écrit par Foued Laroussi, « Mayotte, une île plurilingue en mutation », et l'autre par Gérard Galtier, Les langues africaines, l'éducation et l'édition : suivi du cas de Mayotte. Et, c'est à travers ces deux articles que nous avons eu connaissance de la graphie proposée par Madi.

Selon Galtier (2009: 62-63),

La graphie de Haladi Madi présente quelques particularités : il transcrit les implosives avec des lettres géminées ("bb" et "dd") pour les distinguer des explosives (notées avec des lettres simples "b" et "d") ; il transcrit l'occlusive palatale sonore ([dy] avec la géminée "yy", en faisant ainsi la distinction avec l'occlusive chuintante sonore notée "dj" ; il transcrit la fricative bilabiale sonore avec le digraphe "bv".

Selon Laroussi, le " "e" nasalisé est transcrit avec le "i” par Haladi Madi, alors que le GRPM [Groupe de Recherche sur le Plurilinguisme à Mayotte] a préféré le faire avec un “ê”. » (2009: 33). 


\section{La proposition du GRPM}

Le GRPM ${ }^{1}$ est composé de chercheurs de divers horizons (francophones, spécialistes du shimaoré et shibushi, arabophones et francophones). Le groupe est aussi chargé du projet de recherche sur le plurilinguisme à l'école pour une meilleure maîtrise des langues maternelles à l'école afin de mieux pallier les difficultés d'apprentissage du français sur l'île. Le but du GRPM n'est pas de rajouter un alphabet en plus de celui proposé par l'association SHIME ou le linguistique Haladi Madi,

Mais compte tenu de la polémique autour de cette question et surtout de choix scientifiques différents, nous (le GRPM) avons voulu proposer une solution qui soit fonctionnelle tout en essayant de dépassionner le débat autour de la graphie. Pour cela, nous nous sommes appuyés sur les travaux d'auteurs comoriens, de spécialistes du swahili et d'africanistes de manière générale. Aussi, nous nous sommes inspirés des remarques de Gérald Galtier [...]. Ce dernier rend compte de travaux de linguistes français et comoriens ayant pris des options différentes de celle de SHIME et de Haladi Madi quant à la transcription de certains sons du shimaoré. (Laroussi, 2009 : 32-33).

Le GRPM n'a pas retenu la graphie suggérée par SHIME consistant à transcrire les implosives [b] et [d] par des lettres crossées, parce que, selon Galtier, « cela semble parfaitement inutile et dangereux. Dans le futur, la présence de ces lettres, absentes des claviers courants, sera le plus sûr moyen pour détruire tous les efforts de promotion du shimaoré » (2009 : 62). Le groupe a opté, comme Haladi Madi, pour les géminées "bb et dd" lorsqu'il s'agit de transcrire des implosives et non explosives (choix de Madi). Pour Galtier, ce choix peut s'expliquer par le fait que les sons implosifs sont moins courants dans la langue mahoraise que les consonnes explosives : ainsi, noter ces derniers avec un b et un d standards faciliterait davantage l'écriture et la lecture du shimaoré contrairement aux lettres géminées.

Pour ce qui est de l'occlusive palatale sonore "dy", le GRPM suggère la graphie déjà utilisée par Sophie Blanchy dans son dictionnaire mahorais-français / français-mahorais datant de 1996, c'est- à-dire un d suivit d'un y. Quant à la nasalisation du son [§í], le groupe préfère le transcrire avec "ê" pour des raisons de pratique (Galtier 2009). En résumé, une réelle volonté d’écrire en shimaoré se manifeste mais des divergences de normes sont observées au niveau de la correspondance phonogrammique.

De nos jours, on attend toujours qu'un organisme officiel se prononce sur la question de l'alphabétisation du mahorais. Pour Galtier, l'orthographe des langues de Mayotte (shimaoré et kibushi) reste un problème car elle doit respecter plusieurs contraintes:

Être exacte d'un point de vue scientifique ; permettre un passage aisé vers le français (afin que les enfants puissent étudier ensemble les deux langues à l'école et que les lettrés en français abordent sans difficulté leur langue maternelle); ne pas posséder des caractères phonétiques spéciaux (afin de pouvoir être imprimée sans problème) ; pour le shimaoré, permettre une lecture aisée des textes écrits en swahili et des langues des autres îles comoriennes [... ] ; pour le kibushi, faciliter l'approche des textes écrits en malgache officiel. (Galtier, 2009 : 60).

1. Foued Laroussi est le président du GRPM. 


\section{L'écriture hors-norme : appropriation de la langue mahoraise dans les publicités et les réseaux sociaux}

Dans le cadre de cette étude, notre méthodologie consiste à comparer l'alphabet du shimaoré mise en place par les différents organismes par rapport aux écrits des panneaux publicitaires et ceux produits sur Facebook. Pour cela, nous avons regroupé une trentaine de publicités et une centaine de commentaires sur Facebook, après accord des scripteurs. Nous mettons en italiques les mots ou groupes de mots issus de Facebook ou des panneaux publicitaires; nous indiquons en surligné une transcription correspondant aux préconisations de Madi².

\section{Un hors-norme graphique dû à l'influence du français}

L'influence du français est d'abord marquée au niveau lexical par des emprunts employés dans les énoncés en shimaoré. Parmi ces emprunts, on remarque une recherche d'adaptation : punissiwé pour punir (9 occurrences); filméwa pour filmer (6); histoiri pour histoire (2) ; lineti pour lunettes. Ces mots, recueillis sur Facebook et les panneaux publicitaires, ne sont conformes à aucune norme de l'écrit : utilisant l'alphabet du français et adaptant son lexique (filméwa, punissiwé, histoiri, lineti), ils ne sont conformes ni à sa syntaxe, ni à son lexique ; conformes à la grammaire du shimaoré, leur graphie ne correspond pas exactement à sa prononciation.

Au niveau des graphèmes, on observe des substitutions telles que : ou pour u ; oi pour wa ; é pour e ; cou ss pour s. Ces substitutions montrent que les scripteurs calquent sur le système graphique français pour écrire le shimaoré: par exemple, le scripteur écrit soura («visage »), alors que, selon les normes citées supra, il devrait écrire sura. De même, il écrit voi («il y a ») et non bvwa. D'autres facteurs viennent étayer l'hypothèse d'une influence du français sur le shimaoré comme l'utilisation d'une lettre le «c » qui n'existe pas en shimaoré dans le mot licha (pour lisha) ou la consonne double «ss » dans wassi (« nous ») alors que ce système n'est pas typique de la langue mahoraise mais française. En outre, les scripteurs utilisent l'accent aigu pour le son «é » dans «maoré » (Mayotte) ou dans alors que le shimaoré ne possède pas d'accents graphiques (Association SHIME, 2005 et Laroussi, 2009). Ces interférences produisent des graphies horsnormes.

\section{Segmentation, allongement, aphérèse}

Comme on le constate dans des écrits hors-normes (Steuckardt, ici même), la segmentation des mots n'est pas respectée : ainsi dans muta anlamou pour mutayanlamu ("spécialiste »); wamitsahelewa pour wami tsaelewa («moi, je n'ai absolument pas compris»); koulamtrou pour kula mtru («chaque personne»); kiassina pour kiyasi na (« suffisant pour »). Dans la phrase wami tsayelewa (« moi, je n'ai absolument pas compris »), le scripteur a assemblé le pronom personnel wami (« moi ») avec la forme négative tsayelewa (" je n’ai pas compris »). C'est aussi le cas pour les groupes de mots tels que kula mtru («chaque personne ») où l'adjectif indéfini kula ("chaque ») est agglutiné au nom commun mtru ("personne »); et, dans kiyasina (« suffisant pour »), l'adjectif kiyassi (« suffisant») est lié avec la préposition na (« pour»).

2. Les sons [6] et [d], qui ont une durée accrue (un allongement consonantique) sont notés par des lettres géminées/bb/et/dd/; de même l'occlusive palatale sonore [ß] est notée /yy/. 
D’autres anomalies semblent à mettre en relation avec des effets d'oralité, peut-être recherchés. En vue de marquer un allongement syllabique, les scripteurs ajoutent certaines lettres : hangouu pour hangu ( " chez moi »); hasii babou ya wayidzii pour hasi babu ya wayidzi («à cause des voleurs »); basii d troo pour basi de trwo (" alors bien fait pour toi ») ; kiifou pour kifu (« point ») ; biiga pour binga (« peut-être »). Nous avons ici la répétition de voyelles telles que le « $u$ », le « $i$ » et le « 0 » : nous supposons donc, qu'il s'agit d'un allongement syllabique en vue de marquer l'instance qui ne peut être marqué autrement graphiquement.

On relève également des troncations par aphérèse ont également été relevées ${ }^{3}$. Ainsi, l'aphérèse «za leo » («les nouvelles d'aujourd'hui ») est formée à partir de l'expression « mahabari ya leo ». Signalons que la particule «za », lorsqu'elle est employée dans un domaine, comme la presse dans notre exemple, renvoie à la spécialité du domaine en question. Ainsi « za leo » renvoie aux nouvelles d'aujourd'hui. Autre exemple, si l'on utilise l'expression «za leo » dans le domaine culinaire, il s'agira des plats du jour. De telles graphies, qui rapprochent l'écrit de son oralisation, ont été remarquées dans l'écrit numérique:

le hors-normes tend à rejoindre de nouvelles normes, en cours d'élaboration. Il apparaît en tout cas clairement qu'il y a une méconnaissance de l'alphabet établi du shimaoré car les utilisateurs de Facebook ainsi que les publicitaires emploient des accents qui n'ont pas de correspondance dans la graphie du shimaoré bantu. (Panckhurst, 2009)

En conclusion, le processus de mise à l'écrit du shimaoré a engendré un certain nombre de discussions concernant la correspondance phonogrammique de l'alphabet, ce qui a créé des divergences jusqu'à ce jour. Toutefois, la présence de deux écritures rend possible l'accès à l'écriture du shimaoré (shimaoré francisé vs. shimaoré bantu). En outre, ce débat qui s'est installé dans la société mahoraise démontre une réelle volonté d'écrire des textes en shimaoré, et ce pour des raisons sociétales.

De plus, notre étude a permis de constater que la réflexion sur la standardisation du shimaoré à l'écrit s'oppose nettement au développement d'un shimaoré francisé sur les réseaux sociaux et sur les panneaux publicitaires. Ainsi, les pratiques écrites ordinaires et émergentes montrent en quelque sorte une prédominance des caractéristiques de l'alphabet français (les caractéristiques du shimaoré bantu étant moins utilisées). Aujourd'hui donc, on attend toujours qu'un organisme officiel se prononce sur la question de l'alphabet du shimaoré.

3. L'aphérèse consiste à supprimer des phonèmes ou des syllabes en début de mot ou d'expression. 


\section{Bibliographie}

Ahmed-Changama Mohamed (1997), Dictionnaire français-comorien : dialecte shindzuani, Paris, L'Harmattan, coll. «Archipel des Comores ».

Association SHIME (2006), Narifundrihe shimaore - Apprenons le shimaorais, Mayotte, éd. CDP Mayotte.

Association SHIME (2012), Narifundrihe shimaore - Apprenons le shimaorais, Mayotte, éd. CDP Mayotte.

Blanchy Sophie (1996), Dictionnaire Mahorais français, français mahorais, Paris, L'Harmattan.

Cassagnaud Josy (2007), Mayotte, ces langues qui écrivent ton histoire, Saint-Denis, Connaissances et savoirs.

Galtier Gérard (2009), Les Langues africaines, l'éducation et l'édition suivi du cas de Mayotte, dans Mayotte, une île plurilingue en mutation, F. Laroussi (dir.), Rouen, Presses universitaires de Rouen et du Havre, 49-66.

Laroussi Foued (2009), « Mayotte, une île plurilingue en mutation » dans Mayotte : une île plurilingue en mutation, Presses universitaires de Rouen et du Havre, Mayotte, 11-48.

Madi Haladi (2005), Contribution à l'élaboration d'une description de référence du shimaoré, Thèse de doctorat, Université de Lyon II.

Panckhurst Rachel (2009), "Short Message Service (SMS) : typologie et problématiques futures », T. Arnavielle (dir.), Polyphonies, pour Michelle Lanvin, Montpellier, Université Paul-Valéry Montpellier 3, 33-52.

Rombi Marie-Françoise (1983), Le Shimaore (île de Mayotte, Comores). Première approche diun parler de la langue comorienne, Paris, Peeters-Selaf.

Spelo Rastami (2012), "Propositions pour une graphie normalisée des langues de Mayotte - kibushi et shimaore - en caractères latins », F. Laroussi et F. Liénard (dir.), Plurilinguisme, politique linguistique et éducation: quels éclairages pour Mayotte, Presses de l'Université de Rouen, coll.«Dyalang». 


\section{Annexes}

* Entre parenthèses, notre traduction.

Quelques panneaux publicitaires:

1. Publicité : concessionnaire de voiture

« magari yatriyawo soura maoré »(les plus belles voitures de Mayotte)

2. Publicité : presse écrite

«zaléo » (les nouvelles d'aujourd'hui)

3. Publicité : presse écrite

«malango »

4. Publicité : opticien

« kana matso kadre voihali » (celui qui ne possède pas une bonne vue ne va nulle part)

'mouta anlamou wa lineti de wassi' (les spécialistes des lunettes, c'est nous)

5. Publicité : produits alimentaires

« kima zi shoukou» (les prix ont baissé)

6. Publicité : produits alimentaires

« la Snie a na wassi » (la Snie est avec nous)

7. Publicité : produits alimentaires

«kiassina wami » (bon pour moi)

\section{Quelques publications sur Facebook}

1. KAYLA : amba maskini! d troo !! maoré oho wana damou kasi para tsindzi maoukou koulamtrou asiriya amba avasa vani $d$ hangouu, hasii babou ya wayidzii , basii d troo !! (Genre le pauvre! bien fait pour lui !! à Mayotte les gens ne dorment plus la nuit, les gens ont peur de se faire cambrioler à leur tour, à cause des voleurs, donc bien fait pour lui !!).

2. ALI : who mwuidzi ahou gégé wamitatsahelewa histoiri yavira (c'est un voleur ou je n'ai pas bien compris ce qui s'est passé).

3. SAWDA : Moueeeeeee de toute fason jvai pas gaspiller mn temp sr saaa ... « on va jamais se comprendre jpense » tsi triya kiifou (je m’arrête là).

4. KAMI : Vous pouvez les martiriser, les torturer et on verra si le problème sera résolu hum tsavanou!!!! (Je campe sur mes positions). 
5. SAWDA : C abusé ... c pas que c un voleur qu'il se donne l'utilisation de le filmé sans sn consentement hum «droit d'image » vOi watrou wasoma ari (apparemment, il y a des personnes éduquées soi-disant) sans savoir ce que sa veux dire.. entouka la rOoue tourne.

6. KAYLA : na wa foungwé wa filméwa biinga watso licha wayidzi wa madzi (qu'on les incarcère, qu'on les filme peut-être, ils arrêteront ces voleurs de merde).

7. SAID : jsu sur il é inocent bé voi watrou hun (mais certaines personnes hun (interjection exprimant un ras-le-bol).

8. SAID : javé mm pa remarqer qil lon ataché avec son teeshirt voi madzi soifi (il y a des choses vraiment écœurants).

9. MARIE : c po drole kand il se font arreté mais c drole kand il rentre chez les gens volé ?? pfff. koula atso hibawo na punissiwé mineur ou po. trop c trop watrou wa lemewa (toute personne qui vole doit être punie, mineur ou pas. Trop c'est trop les gens sont fatigués). 